\title{
Electrical Conductivity of Fish Skin Collagen in the Temperature Range 290-380 K
}

\author{
M. GAUZA* AND L. Kubisz \\ Department of Biophysics, Poznań University of Medical Sciences \\ Fredry 10, 61-701 Poznań, Poland
}

\begin{abstract}
Collagen is the major biopolymer of a living organism, which physical properties depend on water content. The observed transmission of spongiform encephalophaties (BSE) to humans resulted in the development of new sources of collagen. Fish skin seems to be one of such safe sources of collagen. Measurements of electrical conductivity as a function of temperature provides information, among other things, on the water release process. Experiment was carried out for fish skin (FSC) collagen (type I) and bovine Achilles tendon collagen (type I). Each sample was heated two times. Current-voltage characteristic was determined for FSC collagen to determine the range, within which the relation is linear. The primary differences appeared to be in electric conductivity, which was higher for FSC collagen than for BAT collagen. Electric conductivity varied from $10^{-6} \mathrm{~S} / \mathrm{m}$ to $10^{-10} \mathrm{~S} / \mathrm{m}$ depending on material and temperature. The process of free and bound water release was manifested as a peak on electrical conductivity-temperature curve between 320-350 K. The activation energy of the charge conduction process, determined on the basis of the Arrhenius plot, was material dependent and considerably higher for FSC collagen.
\end{abstract}

PACS numbers: 72.20.-i, 72.80.Le

\section{Introduction}

Collagen is one of the most important protein of a living organism. The macromolecule of collagen is a complex molecule made of three polypeptide chains, which form the left-turn super helix. Collagen is used in pharmaceutical, cosmetic and food industry, along with medicine and biotechnology. The observed transmission of spongiform encephalophaties (BSE) to humans resulted in an interest in the safety of the bovine derived products. Therefore, development of new sources of collagen is recently observed and fish seems to be one of such safe sources of collagen. Collagen isolated from fish skin by hydration seems to maintain its native structure $[1,2]$. Heating of collagen leads to denaturation, glass transition and water release, which are reflected in changes of electrical conductivity $\sigma[3,4]$. Studies of electric conductivity of proteins indicate, that water facilitates the processes of transport of electric charge carriers $[5,6]$. However, fish skin collagen is the natural protein of better cosmetic specificity even than mammalian collagen, unfortunately, the temperature tolerance of the fish skin collagen (FSC) is limited. Its longer exposition to heat leads to thermal denaturation. Many factors may influence the temperature dependence of collagen electric conductivity, which is sensitive to structural changes, water content and ageing processes. Water release is a complex process, because water molecules participate in

\footnotetext{
* corresponding author; e-mail: mgauza@amp.edu.pl
}

the structure of collagen macromolecule at different ways. In the solid state collagen, water is usually divided into three groups: free water, bound water and structural water [7]. The release of water resulted in the decreased electrical conductivity whereas the temperature increase of collagen resulted in the increase of its electric conductivity. Moreover, changes in the electrical conductivity-temperature relationship can be applied to the evaluation of the influence of different physical and chemical factors on the studied biological material $[8,9]$. The aim of the study was the comparison of the water release process in FSC collagen and bovine Achilles tendon (BAT) collagen on the basis of the temperature dependence of electrical conductivity.

\section{Materials}

The material used in the study was the collagen type I obtained from fish skin by hydration [2]. Collagen gel was poured on the Petri dish and dried at the temperature of $293 \mathrm{~K}$. Next, the cylindrical samples $0.08 \mathrm{~mm}$ thick, $10 \mathrm{~mm}$ in diameter were cut from the film. The collagen type I derived from bovine Achilles tendon (BAT) collagen/SIGMA/was used as the control material. BAT collagen was pressed into tablets, $10 \mathrm{~mm}$ in diameter and $1 \mathrm{~mm}$ thick. The samples were painted on both sides with silver paste electrodes.

\section{Methods}

Measurements of electrical conductivity were carried out at the DC electric field $E=1 \mathrm{kV} / \mathrm{m}$, in the tem- 
perature range of $290-380 \mathrm{~K}$. Samples were placed in the measuring chamber, specially designed, and heated with the heating rate of $1 \mathrm{~K} / \mathrm{min}$, up to the temperature of $380 \mathrm{~K}$. The DC voltage applied to the sample was within the range of voltage-current linearity. Current flowing through the sample was measured by an electrometer. All measurements were performed in air, under atmospheric pressure. The sample was heated from $290 \mathrm{~K}$ to $380 \mathrm{~K}$, maintained at this temperature for one hour to release free and bound water. Next, the sample was cooled to the room temperature and heated again to $380 \mathrm{~K}$. The temperature of a sample was measured by a constantan-copper thermocouple. The values of electrical conductivity $\sigma$ were calculated on the basis of current, voltage applied and the geometrical size of sample, using the following equation:

$$
\sigma=\frac{d I}{S U}
$$

where $S$ is the cross-sectional surface area, $d$ is the thickness of the sample, $U$ is the voltage applied to the sample and $I$ is the measured current. The relative error of the electrical conductivity was less than $\pm 5 \%$.

\section{Results and discussion}

Prior the experiment the current-voltage characteristic was determined for the FSC collagen (Fig. 1). Further measurements were carried out within the range were the Ohm law was obeyed.

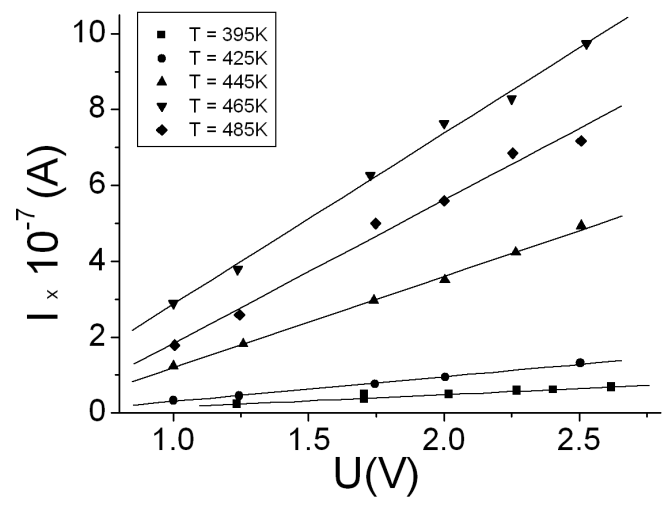

Fig. 1. The current-voltage dependence for FSC collagen.

The first heating run revealed the peak at the temperature range of 320-350 K (Fig. 2a). Samples were kept at $380 \mathrm{~K}$ one hour, than cooled to the ambient temperature and heated again to $380 \mathrm{~K}$. Results obtained during the second heating run, which was carried out at 290-380 K, is shown in Fig. 2b. Instead of the previous peak, the monotonous increase in $\sigma$ was observed. The disappearance of the peak was probably due to the evacuation of free water and bound water, which prior the diffusion out of collagen, was transformed into free water. Similar results were presented in other papers $[10,11]$. Also according to thermogravimetrical studies on dry collagen,

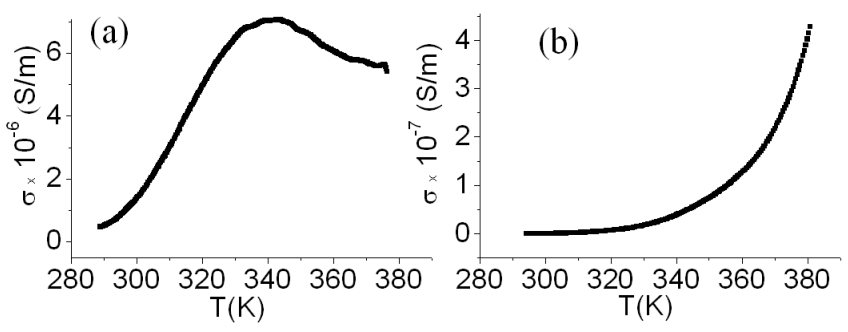

Fig. 2. Temperature dependence of electrical conductivity: (a) during the first heating run; (b) and the second heating run (FSC collagen).

the release of bound water take place in the temperature range $313-443 \mathrm{~K}$, with the highest rate at $353-373 \mathrm{~K}$ [12].

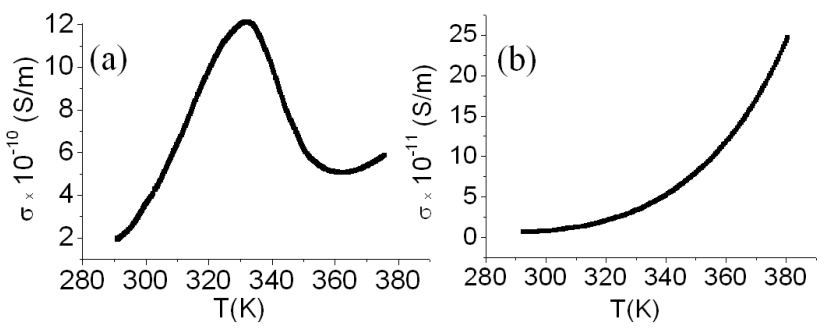

Fig. 3. Temperature dependence of electrical conductivity: (a) during the first heating run; (b) and the second heating run (BAT collagen).

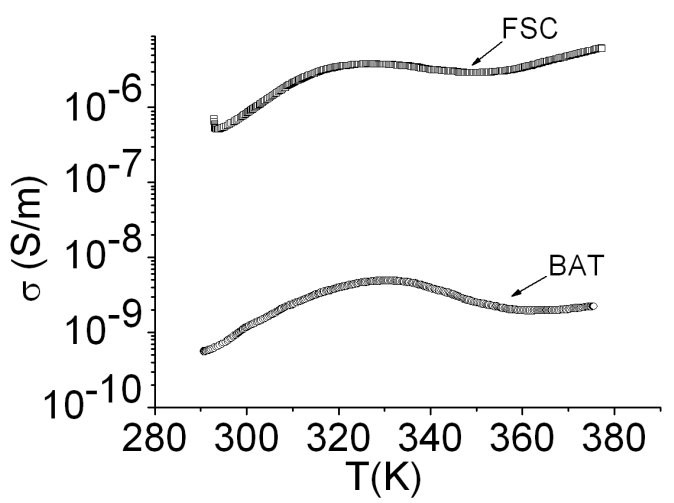

Fig. 4. Temperature dependence of electrical conductivity during the first heating run for FSC collagen and BAT collagen.

Evacuation of free and bound water reduced electrical conductivity of FSC collagen, which varied between $10^{-6} \mathrm{~S} / \mathrm{m}$ during the first heating run and $10^{-7} \mathrm{~S} / \mathrm{m}$ during the second heating run. The primary differences appear to be in electrical conductivity for both materials (Fig. 3) because electrical conductivity of the FSC collagen was higher than the electrical conductivity of the BAT collagen varying from $10^{-9} \mathrm{~S} / \mathrm{m}$ during the first heating run, to $10^{-10} \mathrm{~S} / \mathrm{m}$ during the second heating run. For both materials the characteristic maximum ap- 
peared in the same range of temperature (Fig. 4). Higher electrical conductivity of FSC collagen could be explain either by higher charge carriers mobility or higher charge carriers density in the FSC collagen.

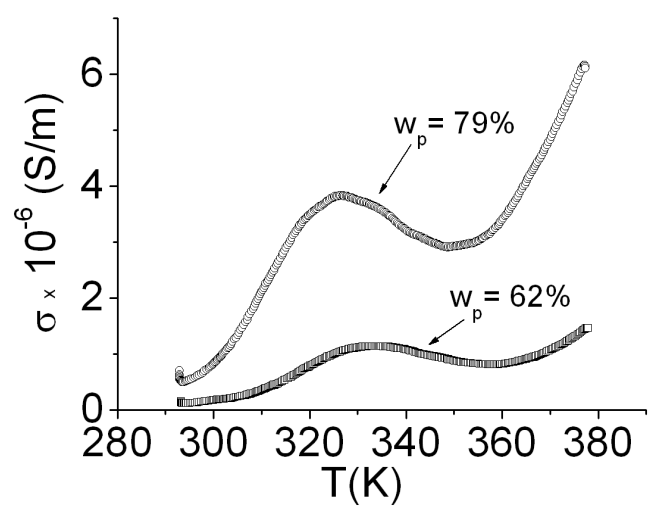

Fig. 5. Temperature dependence of electrical conductivity during the first heating run (FSC collagen).

Since collagen is the hygroscopic material, the effect of air humidity on electrical conductivity was observed as well. The higher the relative humidity of air, at which samples were stored, the higher the peak of electrical conductivity (Fig. 5). The calculated values of conductivity, namely less than $10^{-6} \mathrm{Sm}^{-1}$, the solid state of the samples and low levels of hydration during the second heating - due to the annealing carried out at $380 \mathrm{~K}$ - all imply that the electrode effect can be neglected [13].

On the basis of carried out studies the activation energy $E_{\mathrm{a}}$ of the charge conduction process was determined according to the following formula [14]:

$$
\sigma(T)=\sigma_{0} \exp \left(\frac{E_{\mathrm{A}}}{k T}\right),
$$

where $T$ for temperature, and $k$ for the Boltzmann's constant.

Calculations of activation energy were performed in the intervals of linearity of the following relationship [14]:

$$
\ln \sigma=f\left(\frac{1}{T}\right) .
$$

The correlation coefficient $(r)$ of the above relationship was found to be in the range of 0.99 . The activation energies and appropriate temperature ranges are collected in Table.

TABLE

Range of temperature $(\Delta T)$, activation energy $\left(E_{\mathrm{a}}\right)$ for the first and second heating run.

\begin{tabular}{c|c|c|c|c|c|c|c}
\hline \hline \multicolumn{4}{c|}{ The first heating run } & \multicolumn{3}{c}{ The second heating run } \\
\hline \multicolumn{2}{c|}{ FSC } & \multicolumn{2}{c|}{ BAT } & \multicolumn{2}{c}{ FSC } & \multicolumn{2}{c}{ BAT } \\
\hline$\Delta T$ & $\begin{array}{c}E_{\mathrm{a}} \\
{[\mathrm{K}]}\end{array}$ & $\begin{array}{c}\Delta T \\
{\left[\mathrm{~kJ} \mathrm{~mol}{ }^{-1}\right]}\end{array}$ & $\begin{array}{c}E_{\mathrm{a}} \\
{[\mathrm{K}]}\end{array}$ & $\begin{array}{c}\Delta T \\
{\left[\mathrm{~kJ} \mathrm{~mol}^{-1}\right]}\end{array}$ & $\begin{array}{c}E_{\mathrm{a}} \\
{[\mathrm{K}]}\end{array}$ & $\begin{array}{c}\Delta T \\
{\left[\mathrm{~kJ} \mathrm{~mol} \mathrm{~mol}^{-1}\right]}\end{array}$ & $\begin{array}{c}E_{\mathrm{a}} \\
{[\mathrm{KJ}]}\end{array}$ \\
\hline $294-312$ & $92 \pm 2$ & $294-312$ & $53 \pm 5$ & $300-378$ & $107 \pm 2$ & $300-378$ & $38 \pm 3$ \\
$361-376$ & $51 \pm 10$ & $365-378$ & $16 \pm 2$ & & & &
\end{tabular}

However, activation energies were determined within similar temperature ranges for both materials, activation energy of FSC collagen was always higher than activation energy of BAT collagen. In the case of the temperature range $294-312 \mathrm{~K}$ this difference could be explained by higher humidity of FSC collagen than BAT collagen. Such a difference, due to water level, was found in studies on electrical conductivity of animal bone [15]. Because activation energy of the bound water release in collagen was established as approximately $50 \mathrm{~kJ} \mathrm{~mol}^{-1}[16,17]$, its higher value $\left(92 \mathrm{~kJ} \mathrm{~mol}^{-1}\right)$ in the case of FSC collagen, may indicate simultaneous release of free and bound water (preceded with its transformation to free water) or the contribution of another currently unidentified process.

Activation energy values were lower than $260 \mathrm{~kJ} \mathrm{~mol}^{-1}$, indicating that electrical conductance is not connected with the charge carriers injected from the electrodes [18].

\section{Conclusion}

The temperature dependence of the electric enabled one to observe the release of free and bound water. The water release was followed by the decrease in electrical conductivity for both materials and it appeared in the same temperature range for both, FSC and BAT collagen. The temperature increase of collagen resulted in the increase in its electrical conductivity. Considerable differences were noticed in electrical conductivity, regardless temperature higher electrical conductivity was demonstrated by FSC collagen and activation energy.

\section{Acknowledgments}

Authors deeply appreciate to prof. J. Przybylski for the FSC collagen preparation. 


\section{References}

[1] A. Milan, C. Kristine, K. Heinrich, in: Collagen Hydrolysate and its Relationship to Joint Health, GELITA Health Initiative Berlin, (2004).

[2] J.E. Przybylski, K. Siemaszka-Przybylska, Patent 190737 Urząd Patentu RP, (2002).

[3] V. Samouillan, A. Lamure, E. Maurel, Med. Biol. Eng. Comput. 38, 226 (2000).

[4] S. Matsushita, S. Deki, J. App. Polymer Sci. Vol. 50, 11, 1969 (2003).

[5] D.D. Eley, Semiconducting biological polymers, in: Organic semiconducting polymers, Ed. J.E. Katon, Edward Arnold, London 1968, p. 259.

[6] R. Pething, Ferroelectrics 86, 31 (1988).

[7] S. Nomura, A. Hiltner, J.B. Lando, E. Bear, Biopolymers 16, 231 (1977).

[8] L. Kubisz, E. Marzec, J. Non-Crystal. Solids 305, $322(2002)$.

[9] L. Kubisz, Int. J. Biol. Macromol. 26, 77 (1999).
[10] V.P. Tomaselli, M.H. Shamos, Biopolymers 12, 353 (1973).

[11] L. Kubisz, P. Andrzejewski, J. Biol. Phys. 27, 285 (2001).

[12] A. Bigi, A.M. Fichera, N. Roveri, M.H.J. Koch, Int. J. Biol. Macromol. 9, 176 (1987).

[13] M. Davies, Dielectric Properties and Molecular Behavior, van Nostrand Reinhold, London 1969, p. 280.

[14] R. Pething, Dielectric and electronic properties of biological materials, Wiley\&Sons, Chichester, New York, (1979).

[15] L. Kubisz, The effect of ionizing radiation on electrical and thermal properties of animal bone, $\mathrm{PhD}$ Thesis, Poznań, (2002).

[16] B. Rosenberg, Nature 193, 364 (1962).

[17] M.H. Piner, M. Escoubes, G. Roche, Biopolymers 17, 2799 (1987)

[18] D.D. Eley, in: J.E. Katon (Ed.), Semiconducting Polymers, Marcel Dekker, NewYork 1968, p. 259. 